\title{
Anaphylactic Reactions to Isosulfan Blue Dye During Sentinel Lymph Node Biopsy for Breast Cancer
}

\author{
Tao Wang ${ }^{\mathrm{a}, \mathrm{d}}$, Debin $\mathrm{Xu}^{\mathrm{b}}$, d , Zhen Liao ${ }^{\mathrm{c}, \mathrm{e}}$
}

\begin{abstract}
Background: The sentinel lymph node biopsy is an alternative to axillary dissection for many breast cancer patients. Cases of anaphylactic reaction to the isosulfan blue dye used during sentinel lymph node biopsy have recently been reported. To prospectively reduce the incidence and severity of adverse reactions to isosulfan blue dye, we evaluated the incidence of severe anaphylactic reactions to isosulfan blue dye during the performance of sentinel lymph node biopsy for breast cancer at our institution.
\end{abstract}

Methods: A retrospective chart review study enrolled 1,456 consecutive patients with breast cancer at our institution. Sentinel lymph node biopsy was performed using both isosulfan blue dye and technetium$99 \mathrm{~m}$ sulfur colloid. Cases of anaphylaxis were reviewed in detail.

Results: Overall, $12(0.8 \%)$ of the 1,456 patients had severe anaphylactic reactions. All 12 patients experienced cardiovascular collapse (profound hypotension and tachycardia) and skin reactions; and patients required admission to an intensive care unit bed or equivalent setting for postoperative monitoring. No deaths or permanent disability occurred.

Conclusions: Prompt recognition and aggressive treatment of anaphylactic reactions to isosulfan blue are critical to prevent an adverse outcome. Lymphatic mapping with blue dye should be performed in a setting where personnel are trained to recognize and treat anaphylaxis.

Keywords: Sentinel lymph node; Biopsy; Anaphylaxis

Manuscript submitted August 11, 2018, accepted September 5, 2018

${ }^{a}$ Department of Otolaryngology-Head and Neck Surgery, The Third Affiliated Hospital of Sun Yat-sen University, China

bDepartment of Thyroid and Neck Surgery, The Second Affiliated Hospital of Nan Chang University, China

'Department of Operation Theater Services, Sun Yat-Sen University Cancer Center, State Key Laboratory of Oncology in South China, Collaborative Innovation Center of Cancer Medicine, China

${ }^{\mathrm{d}}$ Equal contributors

${ }^{e}$ Corresponding Author: Zhen Liao, Department of Operation Theater Services, Sun Yat-Sen University Cancer Center, State Key Laboratory of Oncology in South China, Collaborative Innovation Center of Cancer Medicine, 651 Dongfeng Dong Road, Guangzhou 510060, China.

Email: liaozhen@sysucc.org.cn

doi: https://doi.org/10.14740/jcs365w

\section{Introduction}

The sentinel lymph node (SLN) biopsy has replaced axillary lymph node dissection (ALND) as the new standard of care in early breast cancer. Although dissection of the level I and II axillary lymph nodes is the gold standard for axillary staging, there are significant acute and chronic complications associated with the procedure $[1,2]$. Lymphatic mapping and SLN biopsy are now routinely used for staging of clinically lymph node-negative patients with breast cancer. The SLN can be located by intra parenchymal injection of blue dye, either alone or in combination with a radiotracer. Isosulfan blue (IB) is a patent dye, which, after subcutaneous or intra parenchymal injection, is absorbed by lymphoid tissue. It has been increasingly used for lymphatic mapping and for identification of sentinel lymph nodes [3]. Allergic or adverse reactions to IB dye have been reported in 0.06 and $2.7 \%$ of patients undergoing SLN biopsy in 11 single-institution studies representing 22,803 patients, with a mean value of $0.71 \%$ [4]. Symptoms may range from mild (urticaria, erythema) to severe (pulmonary edema, hypotension, vascular collapse). Data on the incidence of severe anaphylactic reactions during the course of SLN biopsy for breast cancer are lacking. Given the substantial number of SLN biopsy currently being performed, even such a small risk of adverse reactions means that a significant number of individuals are at risk. To prospectively reduce the incidence and severity of adverse reactions to IB dye, we evaluated the incidence of severe anaphylactic reactions to IB dye during the performance of SLN biopsy for breast cancer at our institution.

\section{Patients and Methods}

\section{Patient data}

A retrospective chart review study enrolled 1,456 consecutive patients who were admitted to our institution between January 2010 and December 2015 with initial diagnoses of breast cancer without any other previous treatment. Prior informed consent was obtained from patients, as was the approval of the Institutional Research Ethics Committee of the Medical Center. In total, 1,456 patients with breast cancer were eligible for our study received a peritumoral injection with the $99 \mathrm{mTc}$-labeled filtered sulfur colloid. All patients were scheduled to receive IB dye. Lymphatic mapping and SLN biopsy were performed 
as previously described [5].

\section{Results}

Between January 1, 2010 and December 31, 2015, 1,456 patients underwent lymphatic mapping and SLN biopsy. Overall, $12(0.8 \%)$ of the 1,456 patients had severe anaphylactic reactions. All 12 patients experienced cardiovascular collapse (profound hypotension and tachycardia) and skin reactions. In each case, symptoms developed 15 to 30 min after injection of IB dye. All 12 patients required vigorous resuscitation with phenylephrine infusion, antihistamines, steroids, and rapid fluid administration. All 12 patients required admission to an intensive care unit bed or equivalent setting for postoperative monitoring. Three patients had second episodes of anaphylaxis during postoperative monitoring. These allergic reactions consisted of nausea and vomiting, symptoms more consistent with known side effects of narcotic medications than with true allergic reactions. Two patients had a history of true drug allergies: one had a history of mild allergic reactions to penicillin and sulfa drugs, with symptoms including urticaria and itching; and the other had a history of severe anaphylaxis upon exposure to intravenous iodine, with symptoms including bronchospasm and hypotension. No previous exposure to IB dye was reported by any of the 12 patients. No perioperative complications occurred in any of these patients.

\section{Discussion}

Lymphatic mapping and SLN biopsy are now routinely used for staging of clinically node-negative patients with breast cancer. IB is commonly used for lymph node dissection. As experience with SLN biopsy has expanded, reports of adverse reactions to IB dye used in mapping have increased. The incidence of allergic reactions to IB dye ranges from $0.06 \%$ to $2.7 \%$ [4]. Although anaphylaxis was reported with administration of a related dye as early as 1966, anaphylactic reaction to IB has been reported [6]. Montgomery et al had identified three distinct patterns or grades of allergic reaction to the dye [7]. Grade 1 reactions were defined as urticaria or blue hives, pruritis, and/or a generalized rash. Grade 2 reactions were defined as transient hypotension (systolic blood pressure $\geq 70 \mathrm{~mm} \mathrm{Hg}$ ) not requiring vasopressors. Grade 3 reactions were defined as hypotension (systolic blood pressure $<70 \mathrm{~mm} \mathrm{Hg}$ ) requiring vasopressor support. Montgomery reported 39 adverse reactions to IB dye in a series of 2,392 patients (1.6\%) undergoing mapping for breast carcinoma. Nine $(23 \%)$ of the adverse reactions were Grade 3 and three (8\%) were Grade 2 reactions [7]. In a previous report, seven (1.1\%) of 639 patients injected with IB dye during lymphatic mapping for breast carcinoma had severe anaphylactoid reactions to the dye that required vigorous resuscitation [5]. All seven had Grade 3 reactions characterized by cardiovascular collapse requiring vasopressors and admission to an intensive care unit or equivalent setting.

Our patients developed severe anaphylaxis after injection of IB. Low blood pressure required large doses of vasopressors over the first several hours after the event. All patients required vigorous resuscitation with phenylephrine infusion, antihistamines, steroids, and rapid fluid administration. This protracted course of hemodynamic instability may be explained by a continuous systemic uptake of isosulfan dye from the injected site, as was demonstrated by green serum discoloration that lasted throughout the stay in the recovery room. Anaphylaxis represents an immediate type I hypersensitivity reaction, and isosulfan-induced hypersensitivity is an immunoglobulin E-mediated reaction. We could not detect that our patient had previous exposure to isosulfan antigen; however, isosulfan is triphenylmethane dye used in industry to color textiles, cosmetics, detergents, paints, and cold remedies [7]. Therefore, previous exposure to any of these products may have sensitized our patient.

There have been no deaths reported as a result of these systemic reactions. The majority of affected patients stay $24 \mathrm{~h}$ after the procedure. Series have been reported on the use of methylene blue as a substitute for IB [8]. Methylene blue may prove to be less allergenic than IB and therefore remains an intriguing possibility that warrants clinical investigation. Several studies have compared the efficacy of identification of the sentinel node using blue dye to the combination of blue dye and lymphoscintigraphy. In the literature reviewed, no study compared lymphoscintigraphy alone to blue dye, or to a combination of the two. To date there have been no reported allergic complications related to the injection of radioactive isotopes in lymphatic mapping. A well-designed prospective study comparing lymphoscintigraphy alone to the combination may provide evidence on the costs and benefits of using IB. As without any perioperative complication, prevention is often the best management. Perhaps skin testing in these patients will elicit a common antigen or substance that can be eliminated or avoided. Also, identification of people sensitive to the IB dye would allow proper preparation and precautions in these patients to eliminate or limit the extent of their response. Even if we are unable to prevent or eliminate this problem, knowledge of this complication can provide patients with better informed consent and allow breast physicians to be more prepared for these potentially serious reactions.

As its use permeates medicine, more of these allergic reactions should be expected. Although no deaths have been reported and the symptoms and manifestations are reversible, in our study $0.8 \%$ is a significant risk of occurrence. Until a better alternative is proven, high suspicion, early recognition, and appropriate clinical management are recommended. The first line of therapy involves the discontinuation of all anesthetic agents, administration of $100 \%$ oxygen, rapid infusion of large amounts of intravenous fluids, and prompt administration of phenylephrine ( 0.1 to $0.3 \mathrm{mg}$ intravenously given over $10 \mathrm{~min}$ ). The second line of therapy includes H1-blockers (diphenhydramine hydrochloride $50 \mathrm{mg}$ intravenously) and corticosteroids (methylprednisolone $125 \mathrm{mg}$ intravenously). For refractory hypotension in patients receiving beta blockers, glucagon (1-mg ampule) constitutes a third line of treatment.

We chose to use a phenylephrine infusion instead of an epinephrine infusion because of the absence of any changes in airway pressure and because of the presence of sinus tachycardia (which improved after initiating phenylephrine). It is worthwhile mentioning that the stress dose of steroids failed to prevent the anaphylactic response to isosulfan. However, 
preoperative prophylaxis may reduce the severity but not the overall incidence of adverse reactions to IB dye [9].

In breast cancer, the combination of IB dye and a radiotracer has been shown to markedly increase the sensitivity of this procedure. Therefore, we currently advocate the use of IB dye in lymphatic mapping for breast cancer.

\section{Conclusions}

As SLN biopsy rapidly becomes the standard of care for identifying nodal metastases in women with breast cancer, the questions that now the surgeons face relate to the optimal technique and safety. Nonetheless, anaphylactic reactions to IB dye during the course of SLN biopsy for breast cancer, although their incidence is relatively low, could have serious consequences. As part of the informed consent process, patients should be informed of this potentially life-threatening allergic reaction. At a time when so called minimally invasive procedures such as SLN biopsy are shifting towards more cost-effective ambulatory settings, it becomes paramount that the personnel involved in the performance of these procedures be familiar with potential reactions and be prepared to immediately recognize and treat anaphylaxis. Surgeons must know that severe reactions to IB may occur, recognize them early, and be prepared to treat anaphylaxis. However, a larger-scale study on similar lines should be carried out investigating the molecular basis of these adverse reactions.

\section{Funding}

This study was no funds.

\section{Conflict of Interest}

All authors declare that there is no conflict of interest.

\section{References}

1. Kissin MW, Querci della Rovere G, Easton D, Westbury G. Risk of lymphoedema following the treatment of breast cancer. Br J Surg. 1986;73(7):580-584.

2. Larson D, Weinstein M, Goldberg I, Silver B, Recht A, Cady B, Silen W, et al. Edema of the arm as a function of the extent of axillary surgery in patients with stage I-II carcinoma of the breast treated with primary radiotherapy. Int J Radiat Oncol Biol Phys. 1986;12(9):15751582.

3. Coleman RL, Whitten CW, O'Boyle J, Sidhu B. Unexplained decrease in measured oxygen saturation by pulse oximetry following injection of Lymphazurin 1\% (isosulfan blue) during a lymphatic mapping procedure. J Surg Oncol. 1999;70(2):126-129.

4. Bezu C, Coutant C, Salengro A. Anaphylactic response to blue dye during SLN biopsy. Surg Oncol. 2011;20(1):5559.

5. Albo D, Wayne JD, Hunt KK, et al. Anaphylactic reactions to IB dye during SLN biopsy for breast cancer. Am J Surg. 2001;182:393-398.

6. Kopp WL. Anaphylaxis from alphazurine 2G during lymphography. JAMA. 1966;198(6):668-669.

7. Montgomery LL, Thorne AC, Van Zee KJ, et al. IB dye reactions during SLN mapping for breast cancer. Anesth Analg. 2002;95:385-388.

8. Simmons RM, Smith SM, Osborne MP. Methylene blue dye as an alternative to isosulfan blue dye for sentinel lymph node localization. Breast J. 2001;7(3):181-183.

9. Raut CP, Hunt KK, Akins JS, Daley MD, Ross MI, Singletary SE, Marshall GD, Jr., et al. Incidence of anaphylactoid reactions to isosulfan blue dye during breast carcinoma lymphatic mapping in patients treated with preoperative prophylaxis: results of a surgical prospective clinical practice protocol. Cancer. 2005;104(4):692699. 